\title{
The method of surface plasmon-polariton pulses generation via cooperative effects in a waveguide spaser
}

\author{
Alexander Shesterikov ${ }^{1}$, Mikhail Gubin $^{1}$, and Alexey Prokhorov ${ }^{1, *}$ \\ ${ }^{1}$ Vladimir State University named after Alexander and Nikolay Stoletovs, Vladimir, 600000 Russia
}

\begin{abstract}
Cooperative effects arising under conditions of a 0 -D model for a dense ensemble of semiconductor quantum dots located in a dielectric layer near a flat metal surface are considered. The threshold conditions of the effect are determined and the values of the complex refractive index of the dielectric are chosen, which make it possible to increase the efficiency of the formation of surface plasmon-polariton (SPP) pulses.
\end{abstract}

In this paper we consider the problem of SPP pulse generation during the collective exciton decay of excited quantum dots (QDs) with concentration $N$ placed in a dielectric layer near a metal surface (Fig. 1a). The QD size is determined by condition of plasmon-exciton resonance (Fig. 1b), and the QD concentration corresponds to compensation of losses at the considering scales. The development dynamics of collective effects is described by selfconsistent equations for population imbalance $n_{21}$, QD polarization $\rho_{21}$, and Rabi frequency $\Omega_{0}$ :

$$
\begin{gathered}
\dot{\rho}_{12}=-0.5 \cdot \Gamma_{\varepsilon} \rho_{12}+\left(i \Omega_{0}^{*}+i \xi_{0} u_{R} \rho_{12}+\xi_{0} u_{I} \rho_{12}\right) n_{21} \\
\dot{n}_{21}=2 i\left(\Omega_{0} \rho_{12}-\Omega_{0}^{*} \rho_{21}\right)-4 \xi_{0} u_{I}\left|\rho_{12}\right|^{2}-\Gamma_{\varepsilon}\left(1+n_{21}\right), \dot{\Omega}_{0}=-i \cdot t_{R}^{-2} \cdot \rho_{12}-\gamma_{p} \Omega_{0},
\end{gathered}
$$

where $t_{R}=\frac{1}{g \sqrt{N_{a}}}=\sqrt{2 \hbar \varepsilon_{d} \varepsilon_{0} \frac{\partial S_{n}(\bar{\omega})}{\partial \bar{\omega}} /\left(S_{n}(\bar{\omega}) \mu_{12}^{2} N\right)}$ determines the characteristic time for the establishment of quantum correlations between QDs and depends on dipole moment of the interband transition $\mu_{12}$ and the Bergman's parameter $S_{n}(\bar{\omega})=\operatorname{Re}\left(\varepsilon_{d} /\left(\varepsilon_{d}-\varepsilon_{m}(\bar{\omega})\right)\right)$ for the metal-dielectric interface with dielectric permittivities of dielectric $\varepsilon_{d}$ and metal $\varepsilon_{m}(\bar{\omega})=1-\omega_{p}^{2} /\left(\bar{\omega}^{2}+i \gamma_{p} \bar{\omega}\right)$, accounting the electron collision frequency $\gamma_{p}$ and the plasmon frequency $\bar{\omega}=\omega_{p} / \sqrt{2}, \omega_{p}$ is the plasma frequency in metal; $g=\Omega_{0} / \varepsilon$ is the coupling constatnt, $\varepsilon=\sqrt{N_{p}}$, where $N_{p}$ is the number of plasmons in volume $V$. The parameter $\Gamma_{\varepsilon}$ in (1a) and (1b) determines spontaneous rate of losses in QD and

"Corresponding author: avprokhorov33@mail.ru 


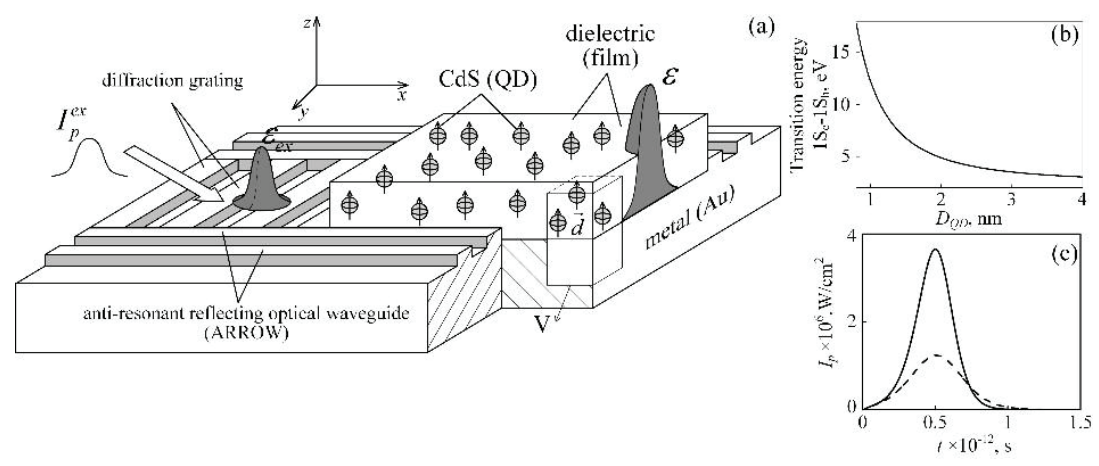

Fig. 1. (a) Formation scheme of SPP pulses in a planar metal/dielectric waveguide [1] pumped by CdS QDs [2]; (b) dependence of the transition energy on the CdS QD size; (c) intensity profiles of SPP pulses (1c) under condition $N=2.83 \cdot 10^{22} \mathrm{~m}^{-3}$ for QDs with $D_{Q D}=1.56 \mathrm{~nm}$ near a gold surface for $\Gamma_{\varepsilon}=6.3 \cdot 10^{11} \mathrm{~s}^{-1}$ (dashed curve) and $\Gamma_{\varepsilon}=0 \mathrm{~s}^{-1}$ (solid curve) decay rates in QD.

$\xi_{0}=N \mu_{12}^{2} / 3 \hbar \varepsilon_{0}$ is associated with accounting the local field of dielectric. The dispersion $u_{R}$ and dissipation $u_{I}$ corrections correspond to the case of dense ensemble of QDs doped in dielectric with refractive index $n=n_{R}+i n_{I}$.

The threshold conditions under which QD concentration proves to be sufficient for the development of collective effects in a waveguide spaser even taking into account fast decay of the SPP in the metal were determined. The comparison of different regimes of SPP pulse formation in a metal/dielectric interface was carried out by use of simulation (1). The optimum values of $n$, which make it possible to completely compensate the relaxation processes in QD (Fig. 1c) were found. Using mean field approximation and assuming $t_{R}<<1 / \gamma_{p}$ the self-consistent problem (1) was reduced to the nonlinear pendulum equation with additional term, which appears due to accounting $u_{I}$ in (1a) and (1b) and provides fast coherent energy exchange between ensemble of QDs and SPP pulse. We also demonstrate the possibility of a strong spectral broadening and generation almost rectangular spectrum SPP pulses due to the existence of a strongly nonlinear near-field effects in the dielectric layer with a dense ensemble of chromophores. However, the possibility of a SPP supercontinuum generation in the system under study requires a substantial reduction in the pulse duration and the use of novel models [3] beyond the slowly-varying-envelope approximation. The possibility of control of SPP pulse dynamic by analogous an effect of triggered superradiance [4], as well as by means of an external magnetic field using magnetic nanostructures [5] is discussed.

The work was supported by RFBR (17-42-330001) and the Ministry of Education and Science of the Russian Federation in the framework of the state task VISU 2017 in the field of scientific research.

\section{References}

[1] A.V. Shesterikov, M.Yu. Gubin, M.G. Gladush, A.V. Prokhorov, JETP 124, 18 (2017)

[2] K.A. Magaryan et al., J. Lumin. 169, 799 (2016)

[3] H. Leblond, P. Grelu, D. Mihalache, Phys. Rev. A 90, 053816 (2014)

[4] P.V. Zinoviev, V.A. Zuikov, A.A. Kalachev et al., Las. Phys. 11, 1307 (2001)

[5] P. Lupo, Z. Haghshenasfard, M.G. Cottam et al., Phys. Rev. B 94, 214431 (2016) 